\title{
Mobile of Point of Sales (POS) Application with Cloud Computing Inventory Management System for Micro and Small Enterprises
}

\author{
Von Kirby German ${ }^{1+}$, Bobby Gerardo ${ }^{2}$ and Bartolome Tanguilig ${ }^{1}$ \\ ${ }^{1}$ Technological Institute of the Philippines, Quezon City, Philippines \\ ${ }^{2}$ West Visayas State University, Iloilo City, Philippines
}

\begin{abstract}
The advancements of computing technologies have resulted to cheaper mobile devices and access to various mobile applications in the market. These developments have also opened opportunities for micro and small enterprises to streamline their business processes without investing heavily on enterprise software licenses and hardware resources. This paper explores the convergence of a mobile point of sales (POS) application and a cloud computing inventory management system. However, one of the major problems in implementing a cloud computing system is the Internet speed in the Philippines. Latency issues can greatly affect the productivity of micro and small businesses since majority of them are engaged in wholesale and retail trade. This can be solved by synchronizing the data coming from the native mobile POS through batch processing with the data of the inventory management system on the cloud in situations where unavailability of, limited, or slow network connection makes it impossible to perform real-time update of data. This paper will also discuss the architecture, data synchronization method, and the challenges and growth prospects of mobile and cloud computing.
\end{abstract}

Keywords: mobile application, cloud computing, data synchronization.

\section{Introduction}

Mobile devices are increasingly becoming an integral part of society as the most effective and convenient productivity and communication tools not bounded by time and place. Annual sales of mobile devices - have outstripped the sale of desktop devices; that occurred more than ten years ago. Driven by information technology (IT) consumerization, the desktop is a staple at home and in the office, but now we live in the post-PC era, with many mobile devices having more computing power that as available on a desktop 15 years ago [1]. Mobile users have a wide variation of services coming from mobile application vendors (e.g., iTunes, Google Play, Windows Store, etc.) which they can use for personal, commercial and business mobile computing purposes.

Cloud computing has created a new way to deliver information system (IS) and information technology. It is a business model that changes how enterprises streamline their processes and increase their efficiency, and as well as improve their cash flow. It provides mobile users with data storage and processing services on a cloud computing platform. Though it is true that information and data on the cloud can be accessed anytime and from anywhere at all, there are times that this system can have serious dysfunction. The cloud computing platform requires a very good and stable Internet connection to be logged on to the server at all times. This will be a major impediment to implement a transaction processing (TP) cloud computing system.

Majority of the micro and small businesses in the Philippines are engaged in wholesale and retail trade that processes day-to-day sales and inventory transactions [2]. A cloud computing platform would be a feasible solution for businesses who have limited time, economic, technical, and operational resources to implement a sales and inventory management system. However, according to the study conducted by Ookla

+ Corresponding author. Tel.: +639088654729.

E-mail address:_vkgconsult@gmail.com. 
last May 2015, the Philippines has the second-slowest Internet in Asia, next only to Afghanistan [3]. This latency issue has a big impact on the operations and productivity of the enterprise, making cloud computing a trend that is still in its infancy in the Philippines today.

This paper will explore the benefits and features of integrating mobile point of sales application--which addresses the latency problem in cloud computing platform, and cloud computing inventory management system for the requirements micro and small enterprises in the Philippines. It will also discuss the synchronization of data between the POS application and the cloud computing system.

\section{Related Works}

Integration of Mobile Point of Sales (POS) Application with Cloud Computing Inventory Management System for Micro and Small Enterprises has been researched for years to achieve an effective alternative way; they gathered some studies related to their proposed mobile application and system.

According to the research study made by Sanjay Ahuja and Alan Rolli entitled "Exploring the Convergence of Mobile Computing with Cloud Computing" (2012), technology advances have made it possible, but desirable to have ubiquitous access to emails and information. More and more individuals are carrying smartphones and utilizing tablet PCs for business and personal use. Mobile cloud computing is predicted to see significant growth through 2015. The iPhone and iPad platform are likely to accelerate the rate of growth of application development. This trend is applicable to other mobile application platforms such as the Android and Windows platforms. Research is continually underway to offer greater mobile abilities such as greater processing speed, longer battery life, greater storage capacity, better display size, and quality. This along with standards and cloud services that support remote data access, storage, and security, is the key to the success of mobile cloud computing.

According to Alexander Stage's research entitled "Synchronization and replication in the context of mobile applications" (2011), mobile computing gives users the chance to access data that is stored in a stationary database or some other data repository at any time and any place. This is also known as ubiquitous data access, which is realized through the use of modern communication links like wireless networks. Typical mobile devices are laptops, but also smaller devices like PDAs or smart cell phones. Mobile computing is regarded by many researchers as the worst case of distributed computing, since it is constrained by many factors that have to be faced and overcome in order to provide reliable and trustworthy applications to end-users.

According to Snehal Warhekar and V. Gaikwad's research entitled "Mobile Computing: Approaches and Issues" (2013), mobile cloud computing is one of mobile technology trends in the future since it combines the advantages of both mobile computing and cloud computing, thereby providing optimal services for mobile users. According to Gartner, corporate employees using smartphones and tablets for business purposes represent about $75 \%$ of the mobile cloud app market.

With regard to the previous study above, another research of Abdullahi Abubakar Imam, Shuib Basr, Rohiza Ahmad entitled "Data Synchronization Between Mobile Devices and Server-side Databases: A Systematic Literature Review" (2015), mobile computing offers the chance to users to access data stored in a data repository or stationary database of the mobile devices at anytime and anywhere. Therefore, it is necessary to establish an effective data sharing and synchronization between these devices and the server with maximum consideration of the following mobile devices limitations: restricted bandwidth of wireless networks, limited resources, e.g. memory, mobility (two types of mobility): terminal (also known as micro) mobility and network (also known as macro) mobility, disconnections, and limited power supply.

\section{Objectives of the Study}

The objective of the researchers when they developed the Mobile Point of Sales (POS) Application and Cloud Computing Inventory Management System is to avoid possible downtime and inability to use the system in cases where it becomes dysfunctional due to network latency issues or slow Internet connectivity, a situation that often is experienced in the Philippines due to poor network implementation by the service providers. This can be achieved by synchronizing the data coming from the native mobile POS through batch 
processing with the data of the inventory management system on the cloud in situations where unavailability of, limited, or slow network connection makes it impossible to perform real-time update of data. This will be a good feature for micro and small enterprises to continuously capture data on POS terminals with or without connectivity and sync them from the mobile POS application with the inventory data of the inventory management system on the cloud at a later time.

\section{System Architecture}

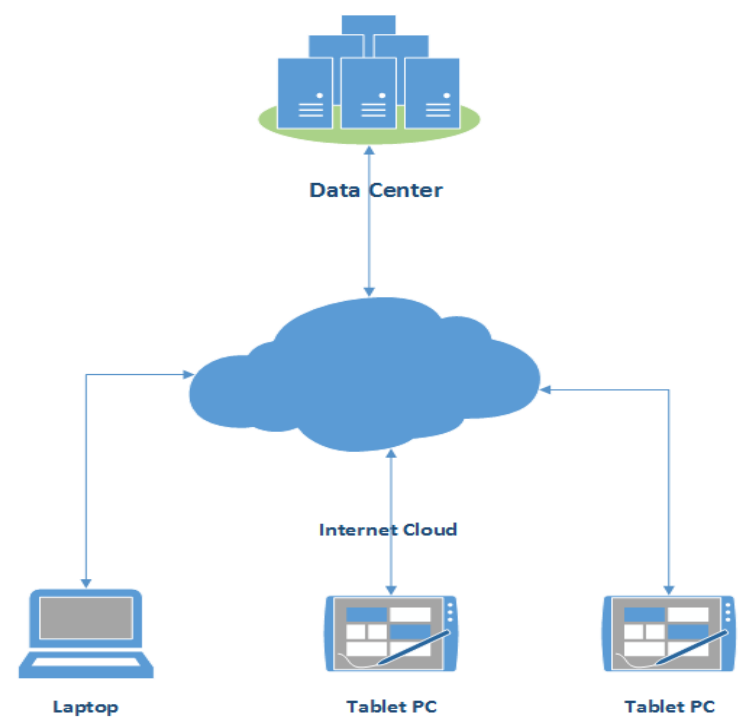

Fig. 1: System architecture.

Fig. 1 illustrates the system architecture showing the mobile POS application installed in the tablet PCs connecting to the Internet, as well as the laptop accessing the inventory management system on the cloud. These application and system are storing and synchronizing their data in the allocated data center.

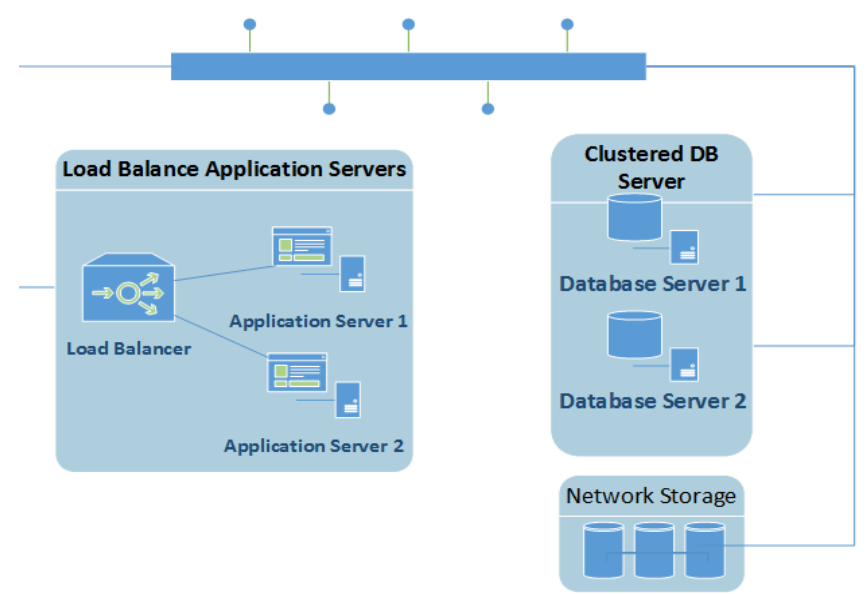

Fig. 2: Physical architecture.

Figure 2 shows the physical architecture which the server application is hosted on two load-balanced application servers which distributes load requests coming from the client. Data is managed in a clustered and redundant database hosts which retrieves and stores data from and to an array of network drives. This architecture ensures performance, high availability and redundancy for the entire system.

Figure 3 presents the different service models, SaaS, PaaS, and IaaS require different share structures of responsibility. Software as a Service (SaaS) gives users the capability to use applications supplied by the service provider but allows no control of the platform or the infrastructure. Platform as a Service (PaaS) gives the capability to deploy consumer-created or acquired applications using programming languages and tools supported by the provider. Infrastructure as a Service (IaaS) allows the user to deploy and run arbitrary software, which can include operating systems and applications [4]. 


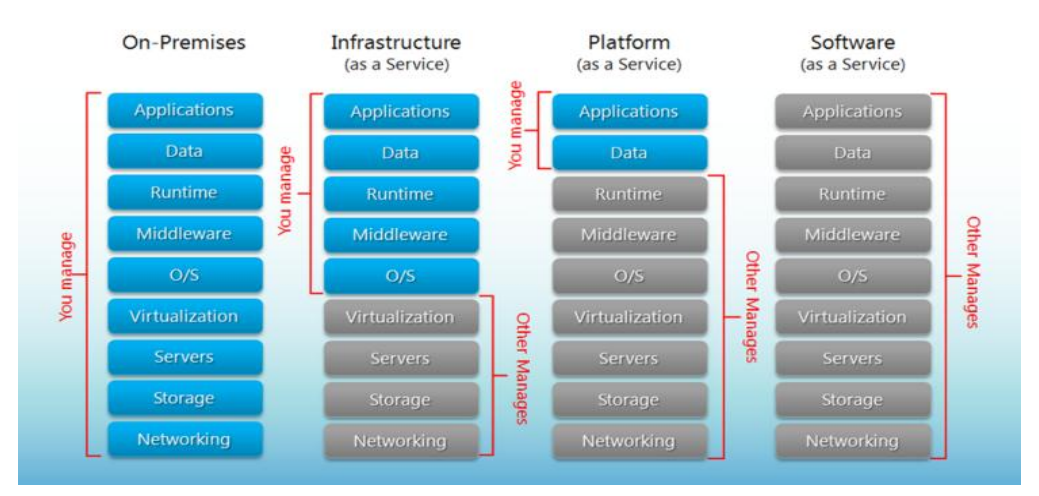

Fig. 3: Separation of responsibilities.

Based on the system and physical architecture, PaaS is the cloud service model that will fit the business requirements of the research project. Platform as a Service offerings facilitate deployment of applications without the cost and complexity of buying and managing the underlying hardware and software and provisioning hosting capabilities, which are suited for micro and small medium enterprises.

\subsection{Data Synchronization}

Data synchronization can be defined as record exchange between two different databases or coherently keeping replicated copies of a dataset [5]. In the context of mobile applications, it is the system that establishes the movement of data between the mobile device and the server-side databases [6]. Database synchronization can be a one-way or a two-way program, and can also be real time or periodic mode, namely Synchronous and Asynchronous [7]. Asynchronous is periodic in nature and happens to be most advantageous for the disconnected environment where clients are allowed to disconnect from the network and still not miss out any item when reconnected. This technique is the most effective way for bandwidth utilization [8].

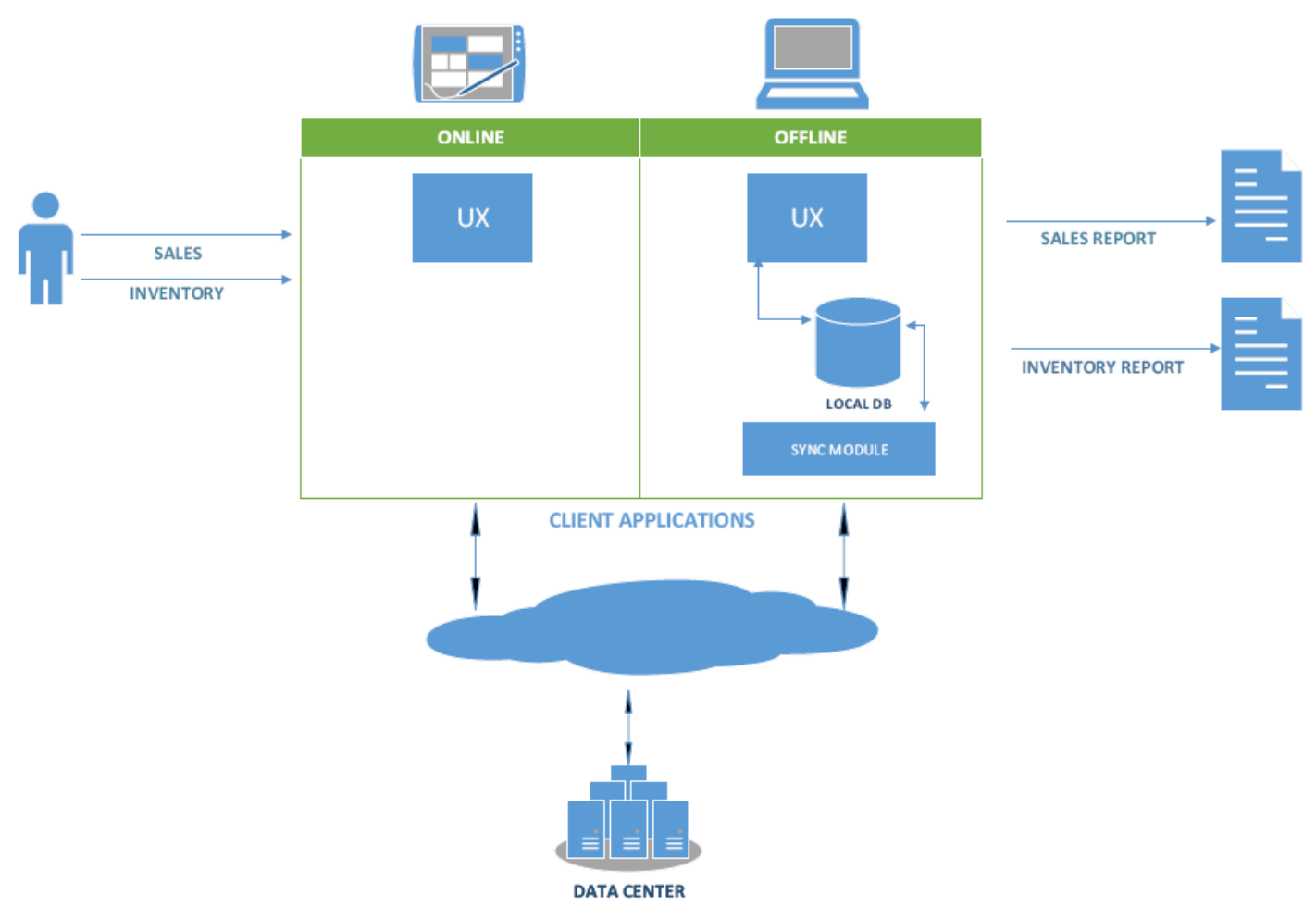

Fig. 4: Framework of network synchronization.

Figure 4 shows a synchronization model in the Database Management System (DBMS) works with the mobile POS application installed in the Tablet PC and the cloud-based inventory management system accessed through a laptop. 
The client application will have an option to perform a synchronous or an asynchronous sync of daily data to the cloud database and this is an option that needs to be toggled by the user.

Synchronous sync can be used when there is a stable connection to the internet. Data will be written directly to the database server as user inputs them on the device.

If there is no stable connection, to user can choose to switch into an offline mode and perform asynchronous sync at a later time. The user inputs the sales transaction on the Tablet PC (e.g. items sold, items returned, etc.), at the end of the day the user will generate the end-of-day sales report and sync it to the cloud computing inventory management system. All data collected throughout the day will be saved into a local database embedded with the client application. The local database allows the client application to have a temporary storage for data without compromising the user experience as it will have the same schema with that of its server counterpart. The user will be able to manage, monitor, and generate reports of the sales and inventory transactions of the business the same way they would in a connected mode. When the time comes that data needs to be synched and a stable connection becomes available, a sync module within the client application will check all changes on the local database and push these changes to the database server. This synchronization can be two-way and should allow for fetching recent data change from the database server as well since the last time the device was online.

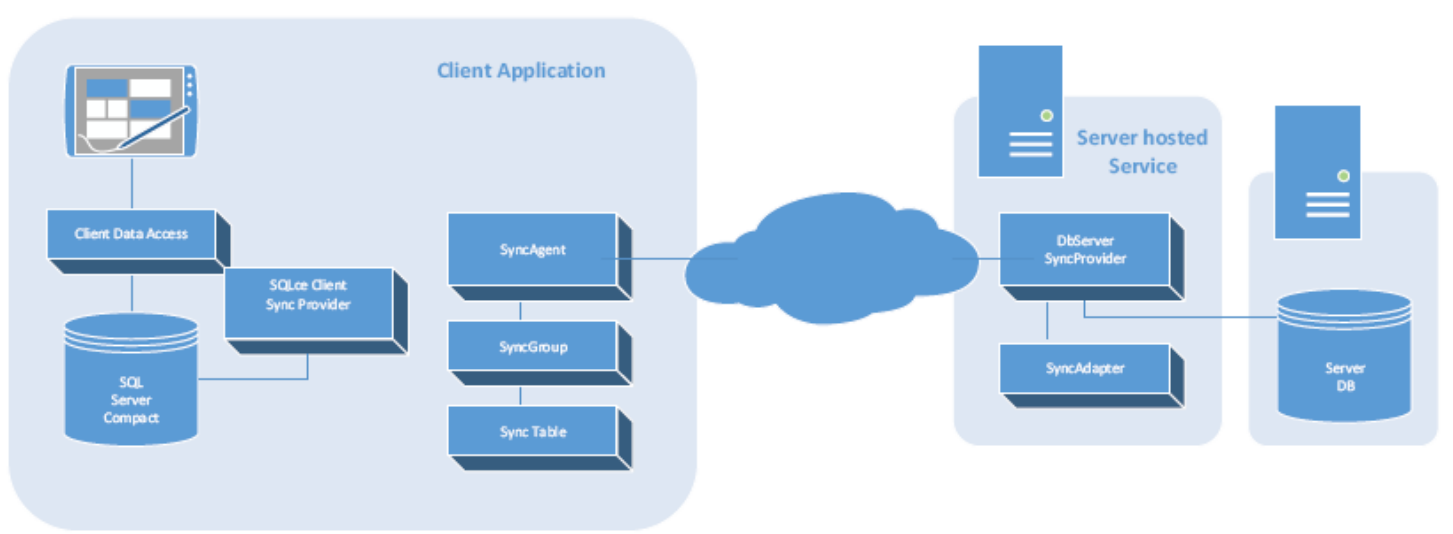

Fig. 5: Sync services in a layered service-oriented architecture.

Figure 5 shows the synchronization method using SQL Server Compact Database. The right portion represents the server-side configuration while the left shows the client side. SQL Server Compact provides a Sync DB Provider for both the server and client sides. All tables to be synched from the client side will be grouped in several sync groups that will go through the client sync DB provider. The receiving server sync DB provider will be configured with several sync adapters to transform the tables coming from the client DB into a schema that is the same with the server DB. All of these features are provided by the Sync Services on SQL Server Compact platform.

\section{Conclusion}

The technological advancements in the areas of computing and mobile technology have paved way to the occurrence of a new computing atmosphere and various powerful mobile devices that changed the landscape of doing business today. Cloud computing is a new business model that created a new way of delivering information system and technology to organizations. Micro and small enterprises can now streamline their business process by automating their day-to-day transactions to increase efficiency and productivity. However, the Philippines being ranked second-slowest in Asia in terms of Internet speed makes cloud computing ineffective to implement to micro and small businesses due to latency issues. Since most of the micro and small enterprises are located in places which have slow or even no Internet connectivity, the work around for this problem is to develop a native mobile Point of Sales application that is integrated with a cloud based inventory management system that can do end of day batch processing data synchronization in case there is no internet connection. The SQL Server Compact platform will be used for the data synchronization. It provides a Sync DB Provider for both the server and client sides. Future enhancements of 
this research paper can explore on data mining using classification methods in developing a decision support system in managing the sales and inventory of the organization.

\section{References}

[1] M. Gendron. Business Intelligence and the Cloud. John Wiley \& Sons, 2014.

[2] SME STATISTICS: 2013 MSME Statistics. Department of Trade and Industry, MSMED Plan, 2011-2016. http://www.dti.gov.ph/dti/index.php/resources/sme-resources/sme-statistics

[3] G. Lasco. The pathetic state of PH Internet. Inquirer.net, September 2015. http://opinion.inquirer.net/88125/thepathetic-state-of-ph-internet

[4] D. Marinescu. Cloud Computing Theory and Prectice. Elsevier Inc, 2013.

[5] D. Sethia, S. Mehta, A. Chodhary, K. Bhatt, S. Bhatnagar. MRDMS-Mobile Replicated Database Management Synchronization. Int. Conf. Signal Process. Integr. Networks. 2014, pp. 624-631.

[6] J. Sedivy, T. Barina, I. MOrozan, A. Sandu. MCsync - Distributed, Decentralized Database for Mobile Devices. IEEE. 2012, pp. 0-5.

[7] L. Zhenyu, C. Zhang, L. Zunfeng. Optimization of Heterogeneous Databases Data Synchronization in WAN by Virtual Log Compression. Futur. Networks, 2010 ICFN '10 Second Int. Conf. 2010, pp.98-101.

[8] A. Imam, S. Basri, R. Ahmad. Data Synchronization Between Mobile Devices and Sever-side Databases: A Systematic Literature Review. Journal of Theoretical and Applied Information Technology, Vol.81, No.2. 2015, pp 364-382. 